\title{
Identificación y virulencia de Metarhizium anisopliae (Hypocreales: Clavicipitaceae) como agente de control biológico de Rhipicephalus microplus (Acari: Ixodidae) en Panamá
}

\author{
Identification and virulence of Metarhizium anisopliae \\ (Hypocreales: Clavicipitaceae) as biological control agent \\ of Rhipicephalus microplus (Acari: Ixodidae) in Panama

\begin{abstract}
Vidal Antonio Aguilera-Cogley1*, Marcelino Jaén-Torrijos ${ }^{2}$, Lissy Yinela Ávila-Rodríguez², José Ángel Herrera-Vásquez ${ }^{1}$, José Natividad Jaén-Sanjur ${ }^{1}$, Anovel Amet Barba-Alvarado ${ }^{1}$
\end{abstract}

\begin{abstract}
RESUMEN
La garrapata Rhipicephalus microplus es el principal ectoparásito del bovino en Panamá y en otras regiones tropicales y subtropicales del mundo. Tradicionalmente, el método de control de $R$. microplus se basa en la utilización de acaricidas químicos. Sin embargo, este método presenta inconvenientes como el desarrollo de resistencia, contaminación al ambiente y a los alimentos, lo que ha promovido la evaluación de estrategias de control alternativas a los acaricidas. El objetivo de este estudio fue identificar y evaluar la virulencia de aislados nativos de Metarhizium anisopliae sobre larvas de R. microplus en condiciones in vitro. La identificación de los aislados Mt1, Mt2 y Mt5 se realizó sobre la base de sus características morfológicas y mediante la secuenciación de la región ITS-5,8S del ADN ribosomal. Los tres aislados nativos se identificaron como M. anisopliae. Se hicieron bioensayos utilizando un diseño experimental completamente al azar. Con cada aislado, se evaluaron cuatro concentraciones de $1 \mathrm{x} 10^{5}, 10^{6}, 10^{7}$ y $10^{8}$ conidias $\mathrm{mL}^{-1}$, y un control (solución de Tween 80 al 0,1\%). Se encontraron diferencias significativas $(\mathrm{P}<0,01)$ en los porcentajes de mortalidad (PM) sobre larvas entre las concentraciones evaluadas para cada uno de los aislados, a los 10, 20 y 30 días postratamiento (DPT). Los tres aislados alcanzaron el máximo PM (> 98\%) sobre larvas a las concentraciones $1 \times 10^{7}$ y $1 \times 10^{8}$ conidias $\mathrm{mL}^{-1}$, a los 30 DPT. En el tratamiento control no se observó mortalidad de larvas. El aislado Mt5 resultó el más virulento con una concentración letal media $\left(\mathrm{CL}_{50}\right)$ de

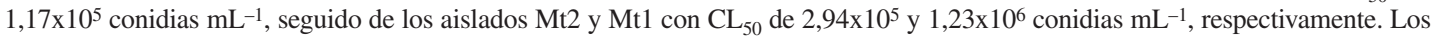
resultados encontrados en el presente estudio sugieren que los aislados nativos Mt5 y Mt2 de M. anisopliae podrían ser considerados agentes promisorios para el control biológico de la garrapata tropical del bovino R. microplus en fincas ganaderas de Panamá.
\end{abstract}

Palabras clave: garrapata, hongos entomopatógenos, región ITS-5,8S, virulencia.

\begin{abstract}
The Rhipicephalus microplus tick is the main ectoparasite of cattle in Panama and other tropical and subtropical regions of the world. Traditionally, the R. microplus control method is based on the use of chemical acaricides. However, this method has obstacles such as the development of resistance, contamination of the environment, and food, which has favored the evaluation of control strategies alternative to acaricides. The objective of this study was to identify and evaluate the virulence of native isolates of Metarhizium anisopliae on larvae of R. microplus under in vitro conditions. The identification of Mt1, Mt2, and Mt5 isolates was performed based on their morphological characteristics and by sequencing the ITS-5.8S region of ribosomal DNA. The three native isolates were identified as M. anisopliae. Bioassays were done using a completely randomized experimental design. With each isolate, four concentrations of $1 \times 10^{5}, 106,107$ and 108 conidia $\mathrm{mL}^{-1}$, and a control $(0.1 \%$ Tween 80 solution) were evaluated. Significant differences $(P<0.01)$ were found in the mortality percentages (PM) on larvae between the concentrations evaluated for each of the isolates, at 10, 20, and 30 days post-treatment $(D P T)$. The three isolates reached the maximum $P M(>98 \%)$ on larvae at concentrations $1 \times 10^{7}$ and $1 \times 108$ conidia $\mathrm{mL}^{-1}$, at $30 \mathrm{DPT}$. In the control treatment, no larval mortality was observed. The Mt5 isolate was the most virulent with a mean lethal concentration $\left(L C_{50}\right)$ of $1.17 \times 105$ conidia $\mathrm{mL}^{-1}$, followed by the Mt 2 and Mt1 isolates with LC50 of $2.94 \times 10^{5}$ and $1.23 \times 10^{6}$ conidia $\mathrm{mL}^{-1}$, respectively. The results found in the present study suggest that the native Mt5 and Mt 2 isolates of M. anisopliae could be considered promising agents for the biological control of the tropical tick of the R. microplus cattle in cattle farms in Panama.
\end{abstract}

Keywords: entomopathogenic fungi, ITS-5.8S region, tick, virulence.

\footnotetext{
1 Grupo de Investigación de Protección Vegetal (GIPV), Centro de Investigación Agropecuaria Divisa (CIAD), Instituto de Investigación Agropecuaria de Panamá (IDIAP), Ctra. Panamericana, Los Canelos, Santa María, Estafeta de Divisa, 0619 Herrera, Panamá.

2 Laboratorio de Salud Animal, Centro de Investigación Agropecuaria Divisa (CIAD), Instituto de Investigación Agropecuaria de Panamá (IDIAP), Ctra. Panamericana, Los Canelos, Santa María, Estafeta de Divisa, 0619 Herrera, Panamá.

* Autor para correspondencia: vidalaguilera@gmail.com, vidal.aguilera@idiap.gob.pa
}

Fecha de Recepción: 13 de Agosto, 2019.

Fecha de Aceptación: 17 de Noviembre, 2019. 


\section{Introducción}

Rhipicephalus microplus (Acari: Ixodidae), conocida como la garrapata tropical del bovino, es el principal ectoparásito del ganado en Panamá (Morán et al., 1995), así como en la mayoría de las regiones tropicales y subtropicales del mundo. Esta garrapata causa graves pérdidas económicas, que se traducen en baja producción de leche, lento crecimiento y transmisión de patógenos, entre estos, Babesia bovis, B. bigemina y Anaplasma marginale (FAO, 1984).

Tradicionalmente, el método de control de $R$. microplus se basa en la utilización de acaricidas, pero su uso excesivo sobre este ectoparásito ha generado la aparición de resistencia química, con el alto costo que esto supone para su control (Kunz y Kemp, 1994; FAO, 2003). En Panamá, en un estudio realizado por Jaén et al. (2015), se mostraron evidencias de la baja sensibilidad de R. microplus a piretroides sintéticos (cipermetrina), en fincas de ganado bovino distribuidas en varias provincias de este país. Esta resistencia química podría traer consigo daños al entorno natural y a la salud humana, por la contaminación de la leche y de la carne bovina (Leal et al., 2003). Por lo tanto, se requiere integrar nuevas opciones de control no químico para minimizar estos efectos nocivos.

Los hongos entomopatógenos son enemigos naturales de artrópodos, y han sido estudiados con éxito a nivel mundial como agentes controladores de la garrapata del bovino (Fernandes y Bittencourt, 2008). El hongo Metarhizium anisopliae (Hypocreales: Clavicipitaceae) ha demostrado ser uno de los entomopatógenos más eficientes para la biorregulación de $R$. microplus en condiciones in vitro e in vivo, contra diferentes estadios de esta garrapata. Además ha supuesto una reducción de fases parasitarias sobre bovinos (Arguedas et al., 2008; Ojeda-Chi et al., 2011; Quinelato et al., 2012).

El control de la garrapata del bovino mediante el uso de hongos entomopatógenos requiere realizar, previamente a las evaluaciones in vitro e in vivo, la correcta identificación del hongo. Tradicionalmente, el método para la identificación de hongos entomopatógenos se basa en la morfología de las esporas, características bioquímicas y propiedades inmunológicas. Sin embargo, técnicas moleculares como la reacción en cadena de la polimerasa (PCR) y el análisis de secuencias de ADN son ampliamente utilizadas para la identificación de diferentes especies de hongos entomopatógenos (Destéfano et al., 2004).

El objetivo del estudio fue identificar aislados nativos de $M$. anisopliae mediante características morfológicas y secuenciación de la región ITS-5.8S del ADN ribosomal y evaluar su virulencia en condiciones in vitro sobre larvas de R. microplus.

\section{Materiales y métodos}

\section{Producción de larvas de Rhipicephalus microplus}

Este estudio se desarrolló en el Centro de Investigación Agropecuaria Divisa (CIAD), del Instituto de Investigación Agropecuaria de Panamá (IDIAP), ubicado en la localidad de Divisa, corregimiento de Los Canelos, distrito de Santa María, provincia de Herrera, Panamá. Inicialmente, se colectaron garrapatas hembras adultas de un bovino confinado en un corral de la Unidad Pecuaria del CIAD sin tratamiento químico previo. Las garrapatas se trasladaron al Laboratorio de Salud Animal del CIAD, donde se desinfectaron con hipoclorito de sodio al $1 \%$ $\mathrm{y}$, seguidamente, se enjuagaron, secaron, pesaron y se revisaron con la ayuda de un estereoscopio SMZ-168 (Motic, CN) para verificar su condición física. La identificación se realizó utilizando la clave taxonómica de garrapatas de BautistaGarfias (2006). Para la producción de huevos, las garrapatas se colocaron en placas de Petri $(90 \mathrm{~mm}$ x $15 \mathrm{~mm}$ ) estériles y se incubaron a $27 \pm 1{ }^{\circ} \mathrm{C}$ y humedad relativa (HR) $\geq 80 \%$ durante 10 días (Jaén et al., 2015). Para la producción de larvas, se tomaron alícuotas de huevos de $50 \mathrm{mg}(\sim 1,000$ huevos), que se colocaron en viales estériles con capacidad para $20 \mathrm{~mL}$, los cuales se taparon con algodón igualmente estéril (Quinelato et al., 2012). La eclosión de las larvas en los viales ocurrió aproximadamente a los 20 días. Se descartaron los viales con porcentajes de eclosión $<95 \%$. En los bioensayos, se utilizaron viales con larvas entre 10 y 15 días de edad.

\section{Identificación morfológica de Metarhizium anisopliae}

Se seleccionaron tres aislados nativos de Metarhizium sp. procedentes de las provincias de Coclé y Herrera, ubicadas en la región central 
de Panamá (Tabla 1), los cuales se encontraban conservados en papel filtro a $-20^{\circ} \mathrm{C}$, en la colección de hongos entomopatógenos del Laboratorio de Protección Vegetal (LPV) del CIAD. Estos hongos se sembraron en placas de Petri que contenían medio de cultivo papa dextrosa agar (PDA) (Difco, USA), y se mantuvieron a $25 \pm 1{ }^{\circ} \mathrm{C}$ en oscuridad durante 14 días. Se obtuvieron cultivos monospóricos de cada uno de los aislados mediante la técnica de diluciones sucesivas (Dhingra y Sinclair, 1995), con la finalidad de obtener cultivos puros para ser usados en la identificación morfológica a nivel de especie, utilizando la clave de identificación de hongos entomopatógenos de Humber (2012). Se evaluó el color de la colonia en medio de cultivo PDA y se determinó la forma, largo y ancho de un total de 25 conidias de cada uno de los aislados con la ayuda de un microscopio óptico DM750 (Leica Mycrosistems, DE), con un aumento de 400X.

\section{Identificación molecular de Metarhizium anisopliae}

La identificación molecular se realizó con los mismos aislados de Metarhizium sp. determinados inicialmente mediante morfología. Para ello, se realizó la extracción del ADN genómico a partir de micelio de cada uno de los aislados, utilizando el kit de extracción EZNA (Omega Bio-tek, USA), según la instrucción del fabricante. La identificación se hizo por PCR, utilizando la pareja de oligonucleótidos ITS1 y ITS4, los cuales amplifican un fragmento de $\sim 500-p b$, correspondiente a la región ITS del ADN ribosómico, incluyendo el gen 5,8S (White et al., 1990). Las concentraciones finales de cada reactivo en la reacción fueron las siguientes: $1 \mathrm{x}$ de tampón PCR, 2,5 mM MgCl $2,0,2$ mM dNTPs (Amresco INC., USA), 0,4 $\mu \mathrm{M}$ de cada cebador, $1 \mathrm{U}$ de Taq ADN polimerasa (Invitrogen, BR), $1 \mu \mathrm{L}$ de cada extracto de ADN, y agua ultrapura estéril hasta alcanzar un volumen de $25 \mu \mathrm{L}$. El ADN se amplificó con la ayuda de un termociclador
Mastercycler® gradient (Eppendorf, USA), el cual se programó para llevar a cabo un ciclo inicial de desnaturalización a $94^{\circ} \mathrm{C}$ durante cuatro minutos, seguido por 35 ciclos de desnaturalización a $94^{\circ} \mathrm{C}$ durante un minuto, hibridación a $55^{\circ} \mathrm{C}$ durante un minuto y extensión a $72{ }^{\circ} \mathrm{C}$ durante un minuto y medio. Adicionalmente, se llevó a cabo un ciclo de extensión final a $72{ }^{\circ} \mathrm{C}$ durante diez minutos, este último con la finalidad de sintetizar todos los fragmentos que posiblemente quedaron incompletos, seguido de un paso de enfriamiento a $4{ }^{\circ} \mathrm{C}$ hasta que las muestras fueron recuperadas. Como control negativo, se utilizó agua ultrapura estéril, la cual se analizó bajo las mismas condiciones descritas anteriormente.

Para confirmar la especie de los aislados de Metarhizium sp., los productos de PCR se purificaron y secuenciaron en Macrogen Inc. en Corea del Sur. Las secuencias se editaron utilizando el programa Chromas versión 2.6.6 (Technelysium Pty Lda, AU), y se depositaron y compararon con las secuencias de referencia existentes en la base de datos del GenBank mediante el programa BLAST del Centro Nacional para la Información Biotecnológica (NCBI, USA) (http://www.ncbi. nlm.nih.gov/). Adicionalmente, se obtuvo un árbol de homología mediante la comparación de las secuencias de los aislados analizados en el presente estudio y de las secuencias de referencia en el programa DNAMAN versión 5.2.9 (Lynnon BioSoft, DE). En este análisis se incluyó como "outgroup" la secuencia del aislado de Beauveria bassiana ARSEF 751 (No. de Accesión AY532045), ya que esta especie de hongo se considera también un agente de control biológico (Rehner y Buckley, 2005).

\section{Preparación de suspensión de conidias}

La cosecha de conidias se realizó a partir de colonias de los aislados de Metarhizium sp. indicados anteriormente, crecidos en medio de

Tabla 1. Aislados nativos de Metarhizium anisopliae, hospedante, origen geográfico y número de accesión de la secuencia de la región ITS-5.8S del ADN ribosomal depositada en el GenBank (NCBI).

\begin{tabular}{lllc}
\hline Aislados & Hospedante & Origen geográfico & No de accesión en el Genbank $^{\text {o }}$ \\
\hline Mt1 & Aeneolamia sp. (Hemiptera: Cercopidae) & Coclé, Panamá & MN294981 \\
Mt2 & Aeneolamia sp. (Hemiptera: Cercopidae) & Coclé, Panamá & MN294982 \\
Mt5 & Cyrtomenus bergi (Hemiptera: Cydnidae) & Herrera, Panamá & MN294983 \\
\hline
\end{tabular}


cultivo PDA y raspadas de su superficie con la ayuda de un escalpelo. Las conidias cosechadas se suspendieron en una solución de Tween 80 al 0,1\%, para luego ser homogenizadas mediante agitación en vortex durante un minuto. La concentración de conidias $\mathrm{mL}^{-1}$ fue estimada con la ayuda de un hemocitómetro y ajustada a las concentraciones de $1 \times 10^{5}, 10^{6}, 10^{7}$ y $10^{8}$ conidias $\mathrm{mL}^{-1}$. La viabilidad de las conidias se determinó colocando $100 \mu \mathrm{L}$ de la suspensión en medio de cultivo PDA e incubada a $25 \pm 1{ }^{\circ} \mathrm{C}$ y HR $\geq 80 \%$. La germinación de las conidias se evaluó después de 24 horas, mediante la observación directa bajo microscopio óptico a 400X, para luego calcular el porcentaje de viabilidad (Ojeda-Chi et al., 2011).

\section{Bioensayos}

En los bioensayos se utilizó un diseño experimental completamente al azar con cuatro tratamientos (concentraciones $1 \times 10^{5}, 10^{6}, 10^{7} \mathrm{y}$ $10^{8}$ conidias $\mathrm{mL}^{-1}$ ) y un control (solución de Tween 80 al 0,1\%). Cada tratamiento se conformó con cuatro repeticiones con viales que contenían las larvas. Los tratamientos se aplicaron siguiendo la metodología propuesta por Quinelato et al. (2012), que consistió en la inyección de $1 \mathrm{~mL}$ de la suspensión de conidias dentro de cada vial para humedecer las larvas durante tres minutos. Seguidamente, se descartó la suspensión de conidias, mientras que los viales se taparon con algodón estéril, incubándolos a $27 \pm 1^{\circ} \mathrm{C}$ y HR $\geq 80 \%$. Como variables de respuesta se evaluó el porcentaje de mortalidad por cada concentración y aislado a los 10, 20 y 30 días postratamiento (DPT) y se estimó la concentración letal media $\left(\mathrm{CL}_{50}\right)$. Al final del experimento, las larvas muertas se colocaron en placas de Petri que contenían medio de cultivo PDA y se incubaron a $25 \pm 1{ }^{\circ} \mathrm{C}$ para inducir el crecimiento del hongo y, posteriormente, confirmar la identidad del hongo entomopatógeno inoculado.

\section{Análisis estadístico}

Los datos de porcentaje de mortalidad a los 10,20 y 30 DPT se analizaron mediante la prueba no paramétrica de Kruskal-Wallis, mientras que la $\mathrm{CL}_{50}$ se obtuvo mediante el análisis Probit. El análisis de los datos se realizó con el software v.3.2.5 (R Core Team, 2016).

\section{Resultados y discusión}

\section{Identificación morfológica y molecular de Metarhizium anisopliae}

Los tres aislados nativos de Metarhizium sp. provenientes de la colección de hongos entomopatógenos del LPV del CIAD mostraron colonias inicialmente de color blanco, que posteriormente se tornaron de color verde olivo. Estos aislados produjeron conidióforos simples con fiálides en forma de botella, mientras que las conidias presentaron forma cilíndrica, aseptada, con dimensiones de 5,60-8,0 × 2,0-2,80 $\mu \mathrm{m}$. Las características morfológicas descritas para los aislados nativos de Metarhizium sp. indicados anteriormente coinciden con las descripciones realizadas por Humber (2012) y Bischoff et al. (2009) para M. anisopliae. La amplificación por PCR de la región ITS-5.8S del ADN ribosómico generó un producto de 490-pb para estos aislados, mientras que las secuencias obtenidas a partir de estos (Tabla 1) mostraron $100 \%$ de homología con secuencias de referencia de los aislados ARSEF 7450 (No. Accesión HQ331464) y ARSEF 7487 (No. Accesión HQ331446) (Figura 1) de M. anisopliae (Schneider et al., 2011). En estudios previos realizados por Driver et al. (2000) y Schneider et al. (2011), la región ITS-5.8S del ADN ribosómico ha sido ampliamente usada para determinar la identidad y diversidad genética de $M$. anisopliae. Basados en las características morfológicas y en el análisis de secuencias de esta región genómica, se

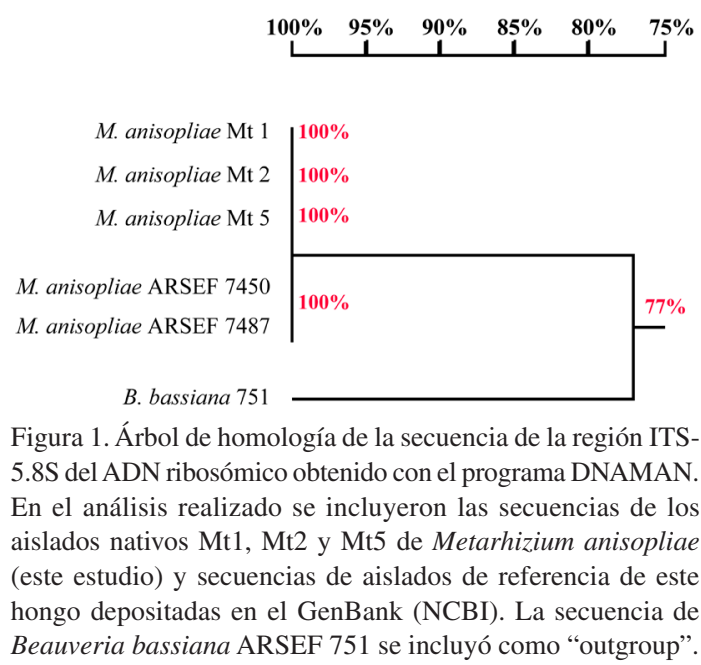


confirmó la identidad de los tres aislados nativos como M. anisopliae.

\section{Bioensayos}

La viabilidad de las conidias resultó $>95 \%$ en los tres aislados de M. anisopliae evaluados. La media de los porcentajes de mortalidad (PM) observada en larvas de $R$. microplus se muestra en la Tabla 2. El análisis estadístico utilizado en el estudio presentó diferencias significativas $(\mathrm{P}<0,01)$ entre las concentraciones evaluadas para cada uno de los aislados de M. anisopliae a los 10, 20 y 30 DPT (Tabla 2). No se observó mortalidad de larvas en el tratamiento control a los 30 DPT. Todas las concentraciones de conidias evaluadas de cada uno de los aislados a los 10 DPT provocaron PM sobre larvas de R. microplus en un rango de 3,5\%-92,3\% (Tabla 2). Estos resultados son similares a los reportados por Fernandes et al. (2004), quienes evaluaron tres aislados de $M$. anisopliae obteniendo un incremento en el PM de larvas entre 11\%-94\% en un rango de concentraciones de $1 \times 10^{5}$ a $1 \times 10^{8}$ conidias $\mathrm{mL}^{-1}$, en comparación con el control (0\%) a los 10 DPT. Los PM sobre larvas aumentaron ligeramente en un rango entre 4,8\%-98\% a los 20 DPT. Los PM más altos, que presentaron valores entre $24 \%-100 \%$, se obtuvieron a los 30 DPT (Tabla 2), observándose PM más elevados ( $>98 \%)$ a las concentraciones de $1 \times 10^{7}$ y $1 \times 10^{8}$ conidias $\mathrm{mL}^{-1}$. Estos resultados son similares a los reportados por Quinelato et al.
(2012), quienes obtuvieron altas mortalidades sobre larvas de R. microplus (> 98\%) a las concentraciones $1 \times 10^{7}$ y $1 \times 10^{8}$ conidias $\mathrm{mL}^{-1}$ de cuatro aislados de M. anisopliae a los 30 DPT. La alta mortalidad inducida por los aislados nativos de M. anisopliae evaluados en el estudio podría ser atribuida a la sensibilidad de la población de $R$. microplus a la infección del hongo. Perinotto et al. (2012) indican que la variación en la virulencia de $M$. anisopliae podría ser atribuida a las diferencias en la susceptibilidad de las poblaciones de garrapatas. Esto sugiere la importancia de considerar poblaciones de garrapatas susceptibles para evaluar la potencial virulencia de hongos entomopatógenos.

$\mathrm{LaCL}_{50}$ se determinó con los datos de mortalidad obtenidos a los 30 DPT (Tabla 3). De acuerdo con los intervalos de confianza al 95\%, el aislado Mt5 de M. anisopliae resultó el más virulento con una $\mathrm{CL}_{50}$ de 1,17 x $10^{5}$ conidias $\mathrm{mL}^{-1}$, seguido del aislado Mt2 con una $\mathrm{CL}_{50}$ de $2,94 \times 10^{5}$ conidias $\mathrm{mL}^{-1}$, mientras que el aislado $\mathrm{Mt1}$ fue el menos virulento con una $\mathrm{CL}_{50}$ de $1,23 \times 10^{6}$ conidias $\mathrm{mL}^{-1}$. En evaluaciones in vitro realizadas por Ángel-Sahagún et al. (2010) sobre larvas de R. microplus, estos autores reportan que los aislados de $M$. anisopliae más virulentos presentaron una $\mathrm{CL}_{50}$ en un rango de $1,1 \times 10^{4}$ y $9,3 \times 10^{5}$ conidias $\mathrm{mL}^{-1}$. Estudios in vitro han demostrado la elevada virulencia de M. anisopliae sobre la garrapata del bovino en diversas etapas de su ciclo de vida (Ấngel-Sahagún et al., 2010; Quinelato et al., 2012; Ren et al., 2012; Fernández-Salas et al., 2017). Por último, se incubaron larvas muertas

Tabla 2. Media de porcentajes de mortalidad de larvas de Rhipicephalus microplus tratadas con aislados nativos de Metarhizium anisopliae.

\begin{tabular}{|c|c|c|c|c|c|c|}
\hline \multirow{2}{*}{ Aislados } & \multirow{2}{*}{$\mathrm{DPT}^{\mathrm{a}}$} & \multirow{2}{*}{ Control } & \multicolumn{4}{|c|}{ Media de mortalidad de Rhipicephalus microplus en larvas $(\%)^{\mathrm{c}}$} \\
\hline & & & $10^{5 \mathrm{~b}}$ & $10^{6}$ & $10^{7}$ & $10^{8}$ \\
\hline \multirow{3}{*}{ Mt1 } & 10 & $0,0 \pm 0,0 \mathrm{a}$ & $3,5 \pm 1,7 \mathrm{~b}$ & $11,3 \pm 6,1 \mathrm{c}$ & $54,3 \pm 15,5 \mathrm{~d}$ & $92,3 \pm 13,5 \mathrm{e}$ \\
\hline & 20 & $0,0 \pm 0,0 \mathrm{a}$ & $4,8 \pm 2,1 \mathrm{~b}$ & $22,0 \pm 11,9 \mathrm{c}$ & $79,5 \pm 19,2 \mathrm{~d}$ & $98,0 \pm 2,9 \mathrm{e}$ \\
\hline & 30 & $0,0 \pm 0,0 \mathrm{a}$ & $24,0 \pm 3,5 b$ & $26,8 \pm 8,9 \mathrm{~b}$ & $98,5 \pm 1,3 \mathrm{c}$ & $99,5 \pm 1,0 \mathrm{c}$ \\
\hline \multirow{3}{*}{ Mt2 } & 10 & $0,0 \pm 0,0 \mathrm{a}$ & $11,0 \pm 3,6 b$ & $28,3 \pm 15,7 \mathrm{c}$ & $35,3 \pm 22,8 \mathrm{c}$ & $89,3 \pm 11,5 \mathrm{~d}$ \\
\hline & 20 & $0,0 \pm 0,0 \mathrm{a}$ & $16,8 \pm 13,5 b$ & $49,3 \pm 18,0 \mathrm{c}$ & $84,8 \pm 10,8 \mathrm{~d}$ & $96,5 \pm 4,5 \mathrm{e}$ \\
\hline & 30 & $0,0 \pm 0,0 \mathrm{a}$ & $26,0 \pm 12,7 b$ & $83,2 \pm 13,6 \mathrm{c}$ & $100,0 \pm 0,0 \mathrm{~d}$ & $100,0 \pm 0,0 \mathrm{~d}$ \\
\hline \multirow{3}{*}{ Mt5 } & 10 & $0,0 \pm 0,0 \mathrm{a}$ & $7,8 \pm 4,2 \mathrm{~b}$ & $38,5 \pm 19,1 \mathrm{c}$ & $58,3 \pm 11,0 \mathrm{~d}$ & $91,5 \pm 10,7 \mathrm{e}$ \\
\hline & 20 & $0,0 \pm 0,0 \mathrm{a}$ & $38,5 \pm 14,2 b$ & $55,3 \pm 15,2 \mathrm{c}$ & $82,3 \pm 5,2 \mathrm{~d}$ & $93,8 \pm 12,5 d$ \\
\hline & 30 & $0,0 \pm 0,0 \mathrm{a}$ & $47,8 \pm 19,2 b$ & $83,0 \pm 12,4 c$ & $99,3 \pm 0,5 \mathrm{~d}$ & $100,0 \pm 0,0 \mathrm{e}$ \\
\hline
\end{tabular}

a DPT: Días postratamiento.

b Concentraciones de conidias $\mathrm{mL}^{-1}$

${ }^{\mathrm{c}}$ Medias dentro de la misma fila con la misma letra no difieren estadísticamente $\mathrm{P}>0.05$ (prueba de Kruskal-Wallis). 
Tabla 3. Concentración letal media $\left(\mathrm{CL}_{50}\right)$ de conidias $\mathrm{mL}^{-1}$ de aislados nativos de Metarhizium anisopliae sobre larvas de Rhipicephalus microplus a los 30 días postratamiento (DPT).

\begin{tabular}{ccc}
\hline Aislados & $\mathrm{CL}_{50}$ & Intervalos de confianza al 95\% \\
\hline Mt1 & $1,23 \times 10^{6}$ & $1,04 \times 10^{6}-1,45 \times 10^{6}$ \\
Mt2 & $2,94 \times 10^{5}$ & $2,53 \times 10^{5}-3,42 \times 10^{5}$ \\
Mt5 & $1,17 \times 10^{5}$ & $8,75 \times 10^{4}-1,56 \times 10^{5}$ \\
\hline
\end{tabular}

de $R$. microplus de los grupos tratados a una HR $\geq 80$, lográndose reaislar los aislados de $M$. anisopliae inoculados inicialmente, lo que confirma la infección y su desarrollo sobre dichas larvas.

\section{Conclusiones}

Este estudio ha permitido identificar aislados nativos de M. anisopliae mediante características morfológicas y el análisis de secuencias de la región ITS-5.8S del ADN ribosomal. Se demostró mediante evaluaciones in vitro la alta virulencia de los aislados Mt5 y Mt2 sobre larvas de $R$. microplus, que los sitúa como potenciales agentes para ser empleados en el control biológico de la garrapata tropical del bovino $R$. microplus en fincas ganaderas de Panamá.

\section{Agradecimiento}

Los autores expresan su agradecimiento al Dr. Luis Galipienso del Instituto Valenciano de Investigaciones Agrarias (IVIA), Valencia, España, por su apoyo en la secuenciación del aislado Mt5 de M. anisopliae. La SENACYT, a través del Sistema Nacional de Investigación (SNI) (subvención 1512018), apoya las actividades de investigación del Dr. José Angel Herrera-Vázquez.

\section{Literatura Citada}

Ángel-Sahagún, C.; Lezama-Gutiérrez, R.; Molina-Ochoa, J.; Pescador-Rubio, A.; Skoda, S.; Cruz-Vázquez, C; Foster, J. 2010. Virulence of Mexican isolates of entomopathogenic fungi (Hypocreales: Clavicipitaceae) upon Rhipicephalus = Boophilus microplus (Acari: Ixodidae) larvae and the efficacy of conidia formulations to reduce larval tick density under field conditions. Veterinary Parasitology, 170: 278-286.

Arguedas, M.; Álvarez, V.; Bonilla, R.

2008. Eficacia del hongo entomopatógeno Metarhizium anisopliae en el control de Boophilus microplus (Acari: Ixodidae). Agronomía Costarricense: Revista de Ciencias agrícolas, 32: 137-147.

Bautista-Garfias, CR.

2006. Entomología Veterinaria Esencial. Ed. INIFAP, México DF, México. 46 p.

Bischoff, J.F.; Rehner, S.A.; Humber, R.A.

2009. A multilocus phylogeny of the Metarhizium anisopliae lineage. Mycologia, 101: 512-530.

Destéfano, R.H.; Destéfano, S.A.; Messias, C.L.

2004. Detection of Metarhizium anisopliae var. anisopliae within infected sugarcane borer Diatraea saccharalis (Lepidoptera, Pyralidae) using specific primers. Genetics and Molecular Biology, 27: 245-252.

Dhingra O.D.; Sinclair, J.B.

1995. Basic plant pathology methods. CRC Press Inc.. Florida, U.S. 448 p.

Driver, F.; Milner, R.J.; Trueman, J.W.

2000. A taxonomic revision of Metarhizium based on a phylogenetic analysis of rDNA sequence data. Mycological Research, 104: 134-150.

Fernandes, E.K.; da Costa, G.L.; Lage de Moraes, A.M.; Bittencourt, V.R.

2004. Entomopathogenic potential of Metarhizium anisopliae isolated from engorged females and tested in eggs and larvae of Boophilus microplus (Acari: Ixodidae). Journal of Basic Microbiology, 44: 270-274.

Fernandes, E.K.; Bittencourt, V.R.

2008. Entomopathogenic fungi against South American tick species. Experimental and Applied Acarology, 46: 71-93.

Fernández-Salas, A.; Alonso-Díaz, M.A.; Alonso-Morales, R.A.; Lezama-Gutiérrez, R., Rodríguez-Rodríguez, J.C.; Cervantes-Chávez, J.A.

2017. Acaricidal activity of Metarhizium anisopliae isolated from paddocks in the Mexican tropics against two populations of the cattle tick Rhipicephalus microplus. Medical and Veterinary Entomology, 31: 36-43.

FAO.

1984. Ticks and tick- borne disease control. A practical field manual. Vol. I. Food and Agricultural Organization. Rome, Italy. 299 p.

FAO.

2003. Resistencia a los antiparasitarios: estado actual con énfasis en América Latina. Food and Agricultural Organization. Rome, Italy. $51 \mathrm{p}$.

Humber, R.A.

2012. Identification of entomopathogenic fungi. In: Lacey, L.A. (Ed.), Manual of Techniques in Insect Pathology, Second ed. Academic Press. California,U.S. pp. 151-187. Jaén, M.; Álvarez, V.; Quintero, R.; Espinales, K.; Rangel, G.; Quintero, N.

2015. Sensibilidad al clorpirifos y cipermetrina en la garrapata Rhipicephalus microplus en fincas ganaderas de Panamá. Revista Ciencia Agropecuaria, 22: 70-77.

Kunz, S.; Kemp, D.

1994. Insecticides and acaricides: resistance and environmental impact. Revue Scientifique et Technique International Office des Epizootics, 13: 1249-1286. 
Leal, A.T.; Freitas, D.R.; da Silva, I.J.

2003. Perspectivas para o controlo do carrapato bovino. Acta Scientiae Veterinariae, 31: 1-11.

Morán, C.; Aparicio, A.; Mora, F.

1995. Programa estacional con uso estratégico de medicamentos y sus componentes, de "Prevalencia de helmintos y control de las garrapatas de los bovinos". Ministerio de Desarrollo Agropecuario. Dirección Nacional de Ganadería. Panamá, pp. 27-38.

Ojeda-Chi, M.M.; Rodríguez-Vivas, R.I.; Galindo-Velasco, E.;

Lezama-Gutiérrez, R.; Cruz-Vázquez, C.

2011. Control de Rhipicephalus microplus (Acari: Ixodidae) mediante el uso del hongo entomopatógeno Metarhizium anisopliae (Hypocreales: Clavicipitaceae): Revisión. Revista Mexicana de Ciencias Pecuarias, 2: 177-192.

Perinotto, W.M.; Angelo, I.C.; Golo, P.S.; Quinelato, S.; Camargo, M.G.; Sá, F.A.; Bittencourt, V.R.

2012. Susceptibility of different populations of ticks to entomopathogenic fungi. Experimental parasitology, 130: 257-260.

Quinelato, S.; Golo, P.S.; Perinotto, W.M.; Sá, F.A.; Camargo, M.G.; Angelo, I.C.; Bittencourt, V.R.

2012. Virulence potential of Metarhizium anisopliae sl isolates on Rhipicephalus (Boophilus) microplus larvae. Veterinary Parasitology, 190: 556-565.
R Core Team.

2016. R: A language and environment for statistical computing. R Foundation for Statistical Computing. Vienna.

Rehner, S.A.; Buckley, E.

2005. A Beauveria phylogeny inferred from nuclear ITS and EF1- $\alpha$ sequences: evidence for cryptic diversification and links to Cordyceps teleomorphs. Mycologia, 97: 84-98.

Ren, Q.; Liu, Z.; Guan, G.; Sun, M.; Ma, M.; Niu, Q.; Li, Y.; Liu, A.; Liu, J.; Yang, J.; Yin, H.; Luo, J.

2012. Laboratory evaluation of virulence of Chinese Beauveria bassiana and Metarhizium anisopliae isolates to engorged female Rhipicephalus (Boophilus) microplus ticks. Biological Control, 63: 98-101.

Schneider, S.; Rehner, S.A.; Widmer, F.; Enkerli, J.

2011. A PCR-based tool for cultivation independent detection and quantification of Metarhizium clade 1. Journal of Invertebrate Pathology, 108: 106-114.

White, T.J.; Bruns, T.; Lee, S.; Taylor, J.W.

1990. Amplification and direct sequencing of fungal ribosomal RNA genes for phylogenetic. In: Innis, M.A.; Gelfand, D.H.; Sninsky, J.J.; White, T.J. (Ed.), PCR protocols, a guide to methods and applications. Academic Press. San Diego, U.S. pp. 315-322. 
